\title{
On the existence of nonnegative radial solutions for $p$-Laplacian elliptic systems
}

\author{
by Daqing Jiang (Changchun) and Huizhao LiU (Harbin)
}

\begin{abstract}
The existence of nonnegative radial solutions for some systems of $m$ $(m \geq 1)$ quasilinear elliptic equations is proved by a simple application of a fixed point theorem in cones.
\end{abstract}

1. Introduction and main result. This paper can be regarded as a continuation of both [2] and [1]. We study the existence of nonnegative radial solutions to the system of $m$ quasilinear elliptic equations

$$
\Delta_{p} u+h(r) f(u)=0, \quad 0<A<r<B,
$$

with one of the following three sets of boundary conditions:

$$
\begin{array}{llll}
u=0 & \text { on } r=A, & \text { and } r=B, \\
u=0 & \text { on } r=A, & \frac{\partial u}{\partial r}=0 & \text { on } r=B, \\
\frac{\partial u}{\partial r}=0 & \text { on } r=A, & u=0 & \text { on } r=B,
\end{array}
$$

where $\left\{x \in \mathbb{R}^{n}: A<r<B\right\}$ is an annulus, $r:=\sqrt{x_{1}^{2}+\ldots+x_{n}^{2}}$, $n \geq 1$, and $\Delta_{p} u:=\left(\operatorname{div}\left|\nabla u_{1}\right|^{p-2} \nabla u_{1}, \ldots, \operatorname{div}\left|\nabla u_{m}\right|^{p-2} \nabla u_{m}\right), h(r) f(u):=$ $\left(h_{1}(r) f_{1}(u), \ldots, h_{m}(r) f_{m}(u)\right), m \geq 1, p>1$.

Throughout this paper, we make the following hypotheses:

$\left(\mathrm{H}_{1}\right) q(t)=\left(q_{1}(t), \ldots, q_{m}(t)\right), q_{j}(t):=L^{p}[w(t)]^{p(n-1) /(p-1)} h_{j}(w(t))$, $j=1, \ldots, m$, is a nonnegative measurable $m$-dimensional vector function defined on $[0,1]$ and satisfies respectively

1991 Mathematics Subject Classification: Primary 34B15.

Key words and phrases: elliptic system, nonnegative radial solution, existence, fixed point theorem in cones.

The work was supported by NNSF of China. 


$$
0<\int_{0}^{1 / 2} G\left(\int_{s}^{1 / 2} q_{j}(t) d t\right) d s+\int_{1 / 2}^{1} G\left(\int_{1 / 2}^{s} q_{j}(t) d t\right) d s<\infty
$$

or

$$
0<\int_{0}^{1} G\left(\int_{s}^{1} q_{j}(t) d t\right) d s<\infty
$$

or

$$
0<\int_{0}^{1} G\left(\int_{0}^{s} q_{j}(t) d t\right) d s<\infty
$$

for $j=1, \ldots, m$, where $G(z):=|z|^{1 /(p-1)} \operatorname{sgn} z$ is the inverse function to $g(y):=|y|^{p-2} y$ for $y, z \in \mathbb{R}$, and $r=w(t)$ is the inverse function to

$$
t=v(r):=\frac{1}{L} \int_{A}^{r} \frac{d s}{s^{(n-1) /(p-1)}}, \quad L:=\int_{A}^{B} \frac{d s}{s^{(n-1) /(p-1)}} .
$$

$\left(\mathrm{H}_{2}\right) f(u)=\left(f_{1}(u), \ldots, f_{m}(u)\right)$ is a nonnegative continuous $m$-dimensional vector function defined on $\mathbb{R}_{+}^{m}$, where $\mathbb{R}_{+}=[0, \infty)$, and satisfying either

(i) $f_{0}=0$ and $f_{\infty}=\infty$ (superlinear), or

(ii) $f_{0}=\infty$ and $f_{\infty}=0$ (sublinear), where

$$
f_{0}:=\lim _{|u| \downarrow 0} \frac{f(u)}{|u|^{p-1}}, \quad f_{\infty}:=\lim _{|u| \uparrow \infty} \frac{f(u)}{|u|^{p-1}} .
$$

Here and henceforth, we denote the norm of $u \in \mathbb{R}^{m}$ by $|u|:=\max \left\{\left|u_{j}\right|\right.$ : $j=1, \ldots, m\}$ and we write $z>y$ (resp. $z \geq y$ ) if $z-y \in \widetilde{\mathbb{R}}_{+}^{m}$ (resp. $\left.z-y \in \mathbb{R}_{+}^{m}\right)$, where $\widetilde{\mathbb{R}}_{+}=(0, \infty)$. Further, we say that a vector $y$ is positive (resp. nonnegative) if $y>0(y \geq 0)$.

The hypothesis $\left(\mathrm{H}_{1}\right)$ allows $q(t) \equiv 0$ on some subintervals of $[0,1]$ and allows a singularity at $t=0$ and $t=1$. For example,

$$
q_{j}(t)=t^{-\alpha_{j}}(1-t)^{-\beta_{j}}(|\cos 2 \pi t|+\cos 2 \pi t), \quad j=1, \ldots, m,
$$

satisfies (1.3) a provided $\alpha_{j}, \beta_{j} \in(0, p)$.

If $u=u(r)$ is a nonnegative radial solution to the problem (1.1)-(1.2), then it satisfies the problem

$$
\left[r^{n-1} g\left(u^{\prime}(r)\right)\right]^{\prime}+r^{n-1} h(r) f(u(r))=0, \quad A<r<B,
$$

with respectively

$$
u(A)=u(B)=0
$$

or

$$
u(A)=u^{\prime}(B)=0
$$


or

$(1.6)_{\mathrm{c}}$

$$
u^{\prime}(A)=u(B)=0,
$$

where $\left[r^{n-1} g\left(u^{\prime}(r)\right)\right]^{\prime}=\left(\left[r^{n-1} g\left(u_{1}^{\prime}(r)\right)\right]^{\prime}, \ldots,\left[r^{n-1} g\left(u_{m}^{\prime}(r)\right)\right]^{\prime}\right)$ and $g(s)=$ $|s|^{p-2} s$ for $s \in \mathbb{R}$.

Let us introduce the change of variables $r=w(t)$ and $y(t):=u(w(t))$, where $w(t)$ is determined by $\left(\mathrm{H}_{1}\right)$. Then the problem $(1.5)-(1.6)$ can be written as

$$
\left[g\left(y^{\prime}(t)\right)\right]^{\prime}+q(t) f(y(t))=0, \quad 0<t<1,
$$

with respectively

$$
y(0)=y(1)=0
$$

or

$$
y(0)=y^{\prime}(1)=0
$$

or

$$
y^{\prime}(0)=y(1)=0,
$$

where $\left[g\left(y^{\prime}(t)\right)\right]^{\prime}=\left(\left[g\left(y_{1}^{\prime}(t)\right)\right]^{\prime}, \ldots,\left[g\left(y_{m}^{\prime}(t)\right)\right]^{\prime}\right)$. Conversely, if $y(t)$ is a nonnegative solution to the problem $(1.7)-(1.8)$, then $u(r):=y(v(r))$ is a nonnegative radial solution to the problem (1.1)-(1.2), where the function $v(r)$ is defined by (1.4). Therefore, we concentrate on the problem (1.7)-(1.8) in the sequel.

We say that a function $y(t)=\left(y_{1}(t), \ldots, y_{m}(t)\right)$ is a nonnegative solution to the problem $(1.7)-(1.8)$ if it satisfies the following conditions:

(i) $y(t) \in C\left([0,1] ; \mathbb{R}_{+}^{m}\right) \cap C^{1}\left((0,1) ; \mathbb{R}^{m}\right)$,

(ii) $y^{\prime}(t)$ is locally absolutely continuous in $(0,1)$, or in $(0,1]$, or in $[0,1)$,

(iii) $\left[g\left(y^{\prime}(t)\right)\right]^{\prime}=-q(t) f(y(t))$ for a.e. $t \in[0,1]$ and $y(0)=y(1)$ or $y(0)=$ $y^{\prime}(1)$ or $y^{\prime}(0)=y(1)=0$.

It is clear that $y(t) \equiv 0$ is a trivial solution to the problem $(1.7)-(1.8)$ when $f(0)=0$. Because of the physical background of the problem above, we are mainly interested in nontrivial nonnegative solutions.

The main purpose of this paper is to extend and improve the existence results of both [1] and [2]. The paper [1] only deals with the case of $m=1$, $p=2$, and [2] only with the case when $m=p=2$ and $f(u)$ is sublinear (i.e. $\left.f_{0}=\infty, f_{\infty}=0\right)$. In [2], some of the conditions on $f(u)$ are superfluous. For brevity, we do not cite other references.

The principal result of this paper is

Theorem 1. Let $\left(\mathrm{H}_{1}\right)$ and $\left(\mathrm{H}_{2}\right)$ hold. Then the problem (1.7)-(1.8) has a nontrivial nonnegative solution $y(t)$, i.e., the problem (1.1)-(1.2) has at 
least one nontrivial nonnegative radial solution on the annulus $\left\{x \in \mathbb{R}^{n}\right.$ : $A<r<B\}$.

The proof will be based on an application of the following fixed point theorem due to Krasnosel'skil (which is quoted from [1]):

Theorem 2. Let $E$ be a Banach space, and let $K \subset E$ be a cone in $E$. Assume $\Omega_{1}, \Omega_{2}$ are open subsets of $E$ with $0 \in \Omega_{1}, \bar{\Omega}_{1} \subset \Omega_{2}$, and let

$$
\Phi: K \cap\left(\bar{\Omega}_{2} \backslash \Omega_{1}\right) \rightarrow K
$$

be a completely continuous operator such that either

(i) $\|\Phi y\| \leq\|y\| \forall y \in K \cap \partial \Omega_{1}$ and $\|\Phi y\| \geq\|y\| \forall y \in K \cap \partial \Omega_{2}$, or

(ii) $\|\Phi y\| \geq\|y\| \forall y \in K \cap \partial \Omega_{1}$ and $\|\Phi y\| \leq\|y\| \forall y \in K \cap \partial \Omega_{2}$.

Then $\Phi$ has a fixed point in $K \cap\left(\bar{\Omega}_{2} \backslash \Omega_{1}\right)$.

2. Some preliminary results. We only consider the problem (1.7)$(1.8)_{\mathrm{a}}$ in this section, since the problems (1.7)-(1.8) $)_{\mathrm{b}}$ and $(1.7)-(1.8)_{\mathrm{c}}$ can be studied in a similar way. To prove the existence of solutions to $(1.7)-(1.8)_{\mathrm{a}}$, we consider the boundary value problem

$$
\left\{\begin{array}{l}
{\left[g\left(w^{\prime}(t)\right)\right]^{\prime}=-q(t) f(y(t)), \quad 0<t<1,} \\
w(0)=w(1)=0
\end{array}\right.
$$

for any $y \in K_{\mathrm{a}}$, where $K_{\mathrm{a}}$ is the cone in $E$ given by

$$
\begin{array}{r}
K_{\mathrm{a}}:=\left\{y \in E: y_{j} \text { is concave on }[0,1]\right. \\
\text { with } \left.y_{j}(0)=y_{j}(1)=0, j=1, \ldots, m\right\},
\end{array}
$$

while $E$ is the Banach space of continuous $m$-dimensional vector functions defined on $[0,1]$ with the norm

$$
\|y\|:=\max \left\{\left\|y_{j}\right\|: j=1, \ldots, m\right\}, \quad\left\|y_{j}\right\|:=\max \left\{\left|y_{j}(t)\right|: 0 \leq t \leq 1\right\} .
$$

It follows that, for any $y \in K_{\mathrm{a}}$, we have

$$
y_{j}(t) \geq\left\|y_{j}\right\| t(1-t) \quad \text { on }[0,1], j=1, \ldots, m .
$$

Lemma 1. For each fixed $y \in K_{\mathrm{a}}$, the boundary value problem (2.1) a has a unique solution $w \in K_{\mathrm{a}}$.

Proof. It is easy to prove the uniqueness. To prove the existence of solutions, we define

$$
w(t)=\left(\Phi_{\mathrm{a}} y\right)(t)=\left(\left(\Phi_{\mathrm{a}} y\right)_{1}(t), \ldots,\left(\Phi_{\mathrm{a}} y\right)_{m}(t)\right)
$$


where

$$
\left.\left(\Phi_{\mathrm{a}} y\right)_{j}(t)\right):= \begin{cases}\int_{0}^{t} G\left(\int_{s}^{\sigma_{j}} q_{j}(r) f_{j}(y(r)) d r\right) d s, & 0 \leq t \leq \sigma_{j}, \\ \int_{t}^{1} G\left(\int_{\sigma_{j}}^{s} q_{j}(r) f_{j}(y(r)) d r\right) d s, & \sigma_{j} \leq t \leq 1,\end{cases}
$$

for $y \in K_{\mathrm{a}}, j=1, \ldots, m$, where $\sigma_{j}$ is a solution of the equation

$$
\begin{aligned}
z_{0}^{(j)}(\tau) & :=\int_{0}^{\tau} G\left(\int_{s}^{\tau} q_{j}(r) f_{j}(y(r)) d r\right) d s \\
& =\int_{\tau}^{1} G\left(\int_{\tau}^{s} q_{j}(r) f_{j}(y(r)) d r\right) d s:=z_{1}^{(j)}(\tau), \quad 0 \leq \tau \leq 1 .
\end{aligned}
$$

Obviously, for each $j \in\{1, \ldots, m\}$ the above equation has a solution $\sigma_{j} \in(0,1)$, since $z_{0}^{(j)}(\tau)$ is a nondecreasing continuous function defined on $[0,1]$ with $z_{0}^{(j)}(0)=0$ and $z_{1}^{(j)}(\tau)$ is a nonincreasing continuous function defined on $[0,1]$ with $z_{1}^{(j)}(1)=0$. Furthermore, if $\sigma_{j}, \delta_{j} \in(0,1), \delta_{j}>\sigma_{j}$, are two solutions, then

$$
q_{j}(t) f_{j}(y(t)) \equiv 0 \quad \text { for almost all } t \in\left[\sigma_{j}, \delta_{j}\right],
$$

which implies that $w_{j}(t)=\left(\Phi_{a} y\right)_{j}(t) \equiv w_{j}\left(\sigma_{j}\right)$ on $\left[\sigma_{j}, \delta_{j}\right]$. This shows that $w$ is well defined, and so is $\Phi$.

From the definition of $w$ and $\Phi$ for $y \in K_{\mathrm{a}}$ we have $(j=1, \ldots, m)$

(i) $w=\Phi y \in K_{\mathrm{a}},\left\|w_{j}\right\|=w_{j}\left(\sigma_{j}\right)$,

(ii) $w_{j}^{\prime}(t)= \begin{cases}G\left(\int_{t}^{\sigma_{j}} q_{j}(r) f_{j}(y(r)) d r\right) \geq 0, & 0<t \leq \sigma_{j}, \\ -G\left(\int_{\sigma_{j}}^{t} q_{j}(r) f_{j}(y(r)) d r\right) \leq 0, & \sigma_{j} \leq t<1,\end{cases}$

(iii) $\left[g\left(w_{j}^{\prime}(t)\right)\right]^{\prime}=-q_{j}(t) f_{j}(y(t))$ a.e. in $(0,1)$, and $w_{j}(0)=w_{j}(1)$.

This shows that $w(t)$ is a solution to $(2.1)_{\mathrm{a}}$ and a concave function defined on $[0,1]$. Now the lemma is proved.

By Lemma 1, we get

Lemma 2. Let $P(t)$ be a solution to problem $(2.1)_{\text {a }}$ with $f \equiv 1$. Then $P_{j}(t) \leq P_{j}\left(\sigma_{j}^{*}\right)(j=1, \ldots, m)$ where $\sigma_{j}^{*}$ is a solution of the equation

$$
\int_{0}^{\tau} G\left(\int_{s}^{\tau} q_{j}(r) d r\right) d s=\int_{\tau}^{1} G\left(\int_{\tau}^{s} q_{j}(r) d r\right) d s, \quad j=1, \ldots, m .
$$


Lemma 3. Let $w(t)$ be a solution to problem $(2.1)_{\mathrm{a}}$ with $f_{j}(y) \leq M^{p}(j=$ $1, \ldots, m)$. Then $w(t) \leq M P(t)$, i.e., $(\Phi y)(t) \leq M P(t)$.

Proof. Let $z(t)=w(t)-M P(t)$. If the lemma were not true, there would exist a $j \in\{1, \ldots, m\}$ and an interval $\left(t_{1}, t_{2}\right) \subset(0,1)$ such that $z_{j}(t)>0$ in $\left(t_{1}, t_{2}\right)$ and $z_{j}\left(t_{1}\right)=z_{j}\left(t_{2}\right)=0$. Notice that

$$
\left[g\left(w_{j}^{\prime}(t)\right)\right]^{\prime}=-q_{j}(t) f_{j}(y(t)) \geq-M^{p} q_{j}(t)=\left[g\left(M P_{j}^{\prime}(t)\right]^{\prime} \quad \text { a.e. in }\left(t_{1}, t_{2}\right),\right.
$$

i.e., $z_{j}^{\prime}(t)>0$ in $\left(t_{1}, t_{2}\right)$. This implies that $0=z_{j}\left(t_{1}\right)<z_{j}\left(t_{2}\right)=0$, which is a contradiction. The lemma is proved.

Lemma 4. For any bounded and closed $D \subset K_{\mathrm{a}}$, the set $\Phi(D)$ is equicontinuous on $[0,1]$.

Proof. Let $M^{p}=\sup \left\{f_{j}(y): y \in D, j=1, \ldots, m\right\}$. For any $\varepsilon>0$, from the continuity of $P(t)$ on $[0,1]$ and $P(0)=P(1)=0$, it follows that there is a $\delta_{1} \in(0,1 / 4)$ such that

$$
P_{j}(t)<\varepsilon /(2 M) \quad \text { for } t \in\left[0,2 \delta_{1}\right] \cup\left[1-2 \delta_{1}, 1\right], j=1, \ldots, m .
$$

By Lemma 3,

$(\Phi y)_{j}(t) \leq M P_{j}(t)<\varepsilon / 2 \quad$ for $t \in\left[0,2 \delta_{1}\right] \cup\left[1-2 \delta_{1}, 1\right], j=1, \ldots, m$.

Let $j \in\{1, \ldots, m\}$ be fixed. If $(\Phi y)_{j}\left(\sigma_{j}\right)<\varepsilon$, then for any $t_{1}, t_{2} \in[0,1]$,

$$
\left|(\Phi y)_{j}\left(t_{1}\right)-(\Phi y)_{j}\left(t_{2}\right)\right| \leq\left|(\Phi y)_{j}\left(\sigma_{j}\right)-(\Phi y)_{j}(0)\right|<\varepsilon .
$$

If $(\Phi y)_{j}\left(\sigma_{j}\right) \geq \varepsilon$, then $\sigma_{j} \in\left[2 \delta_{1}, 1-2 \delta_{1}\right]$ and hence for $t \in\left[\delta_{1}, 1-\delta_{1}\right]$,

$$
\left|(\Phi y)_{j}^{\prime}(t)\right|=\left|G\left(\int_{t}^{\sigma_{j}} q_{j}(r) f_{j}(y(r)) d r\right)\right| \leq M G\left(\int_{\delta_{1}}^{1-\delta_{1}} q_{j}(r) d r\right) \leq L .
$$

Put $\delta_{2}=\varepsilon / L$. Then for $t_{1}, t_{2} \in\left[\delta_{1}, 1-\delta_{1}\right]$ with $\left|t_{1}-t_{2}\right|<\delta_{2}$ we have

$$
\left|(\Phi w)_{j}\left(t_{1}\right)-(\Phi w)_{j}\left(t_{2}\right)\right| \leq L\left|t_{1}-t_{2}\right|<\varepsilon .
$$

Set $\delta=\min \left\{\delta_{1}, \delta_{2}\right\}$. Then for $t_{1}, t_{2} \in[0,1]$ with $\left|t_{1}-t_{2}\right|<\delta$ it follows that

$$
\left|(\Phi w)_{j}\left(t_{1}\right)-(\Phi w)_{j}\left(t_{2}\right)\right|<\varepsilon .
$$

This shows that $\Phi(D)$ is equicontinuous on $[0,1]$.

Lemma 5. The mapping $\Phi$ restricted to $K_{\mathrm{a}}$ is continuous.

Proof. Assume that $\left\{y^{\{k\}}\right\}_{k=0}^{\infty} \subset K_{\mathrm{a}}$ and $y^{\{k\}}(t)$ converges to $y^{\{0\}}(t)$ uniformly on $[0,1]$. By Lemmas 3 and $4,\left\{\Phi y^{\{k\}}(t)\right\}_{k=1}^{\infty}$ is uniformly bounded and equicontinuous on $[0,1]$. By the Arzelà-Ascoli Theorem, there exist uniformly convergent subsequences in $\left\{\Phi y^{\{k\}}(t)\right\}_{k=1}^{\infty}$. Let $\left\{\Phi y^{\{k(i)\}}(t)\right\}_{i=1}^{\infty}$ be a subsequence which converges to $v(t)=\left(v_{1}(t), \ldots, v_{m}(t)\right)$ uniformly 
on $[0,1]$ and $\left\{\sigma^{\{k(i)\}}\right\}_{i=1}^{\infty}$ converges to $\bar{\sigma}=\left(\bar{\sigma}_{1}, \ldots, \bar{\sigma}_{m}\right)$. Then there exists an $H>0$ such that

$$
\left\|y^{\{k(i)\}}\right\| \leq H,
$$

and hence there exists an $M>0$ such that $f_{j}\left(y^{\{k(i)\}}\right) \leq M^{p}, j=1, \ldots, m$. By Lemma 3 it follows that

$$
\left\|\Phi y^{\{k(i)\}}\right\| \leq M\|P\| .
$$

Inserting $y^{\{k(i)\}}$ and $\sigma^{\{k(i)\}}=\left(\sigma_{1}^{\{k(i)\}}, \ldots, \sigma_{m}^{\{k(i)\}}\right)$ into (2.4), (2.5) and then letting $i \rightarrow \infty$, for $j=1, \ldots, m$ we obtain

$$
v_{j}(t)= \begin{cases}\int_{0}^{t} G\left(\int_{s}^{\bar{\sigma}_{j}} q_{j}(r) f_{j}\left(y^{(0)}(r)\right) d r\right) d s, & 0 \leq t \leq \bar{\sigma}_{j}, \\ \int_{t}^{s} G\left(\int_{\bar{\sigma}_{j}}^{s} q_{j}(r) f_{j}\left(y^{(0)}(r)\right) d r\right) d s, & \bar{\sigma}_{j} \leq t \leq 1,\end{cases}
$$

and

$$
\begin{aligned}
v_{j}\left(\bar{\sigma}_{j}\right) & =\int_{0}^{\bar{\sigma}_{j}} G\left(\int_{s}^{\bar{\sigma}_{j}} q_{j}(r) f_{j}\left(y^{(0)}(r)\right) d r\right) d s \\
& =\int_{\bar{\sigma}_{j}}^{1} G\left(\int_{\bar{\sigma}_{j}}^{s} q_{j}(r) f_{j}\left(y^{(0)}(r)\right) d r\right) d s .
\end{aligned}
$$

Here we have applied the Lebesgue Dominated Convergence Theorem, since $f_{j}\left(y^{\{k(i)\}}\right) \leq M^{p}$ for every $j=1, \ldots, m$. From the definition of $\Phi$, we know that $v(t)=\left(\Phi y^{\{0\}}\right)(t)$ on $[0,1]$. This shows that each subsequence of $\left\{\Phi y^{\{k\}}(t)\right\}_{k=1}^{\infty}$ uniformly converges to $\left(\Phi y^{\{0\}}\right)(t)$ on $[0,1]$. Therefore the sequence $\left\{\Phi y^{\{k\}}(t)\right\}_{k=1}^{\infty}$ itself uniformly converges to $\left(\Phi y^{\{0\}}\right)(t)$ on $[0,1]$. This means that $\Phi$ is continuous at $y^{\{0\}} \in K_{\mathrm{a}}$. Therefore $\Phi$ is continuous on $K_{\mathrm{a}}$, since $y^{\{0\}} \in K_{\mathrm{a}}$ is arbitrary.

Combining Lemmas 1-5 we have

Lemma 6. $\Phi: K_{\mathrm{a}} \rightarrow K_{\mathrm{a}}$ is completely continuous.

3. Proof of Theorem 1. We begin by proving the existence of nontrivial nonnegative solutions to the problem (1.7)-(1.8)a .

First suppose that $f_{0}=0$ and $f_{\infty}=\infty$. By the assumption $\left(\mathrm{H}_{2}\right)(\mathrm{i})$, we can choose a $\varrho_{1}>0$ such that

$$
f_{j}(u) \leq(\varepsilon|u|)^{p-1} \quad \text { whenever } 0 \leq|u| \leq \varrho_{1}, j=1, \ldots, m,
$$

where $\varepsilon>0$ satisfies the condition 


$$
\begin{aligned}
0<\varepsilon \int_{0}^{1 / 2} G\left(\int_{s}^{1 / 2} q_{j}(t) d t\right) d s & \\
& +\varepsilon \int_{1 / 2}^{1} G\left(\int_{1 / 2}^{s} q_{j}(t) d t\right) d s<1, \quad j=1, \ldots, m .
\end{aligned}
$$

Thus, if $y \in K_{\mathrm{a}}$ with $\|y\|=\varrho_{1}$, then it follows from (3.1) and (3.2) that

$$
\begin{aligned}
\left\|w_{j}\right\| & =\int_{0}^{\sigma_{j}} G\left(\int_{s}^{\sigma_{j}} q_{j}(t) f_{j}(y(t)) d t\right) d s=\int_{\sigma_{j}}^{1} G\left(\int_{\sigma_{j}}^{1} q_{j}(t) f_{j}(y(t)) d t\right) d s \\
& \leq \varepsilon\|y\|\left[\int_{0}^{1 / 2} G\left(\int_{s}^{1 / 2} q_{j}(t) d t\right) d s+\int_{1 / 2}^{1} G\left(\int_{1 / 2}^{s} q_{j}(t) d t\right) d s\right] \\
& <\|y\| \quad \text { whenever either } \sigma_{j} \geq 1 / 2 \text { or } \sigma_{j} \leq 1 / 2, j=1, \ldots, m,
\end{aligned}
$$

i.e.

$$
\left\|\Phi_{a} y\right\|<\|y\| \quad \forall y \in K_{\mathrm{a}} \cap \partial \Omega_{1},
$$

where $\Omega_{1}:=\left\{y \in E:\|y\|<\varrho_{1}\right\}$ and $\Phi_{\mathrm{a}}$ and $K_{\mathrm{a}}$ are defined as in Section 2.

From $(1.3)_{\mathrm{a}}$, we know that there exists a $\delta \in(0,1 / 4)$ such that

$$
\begin{aligned}
\min \left\{\int_{\delta}^{1 / 2} G\left(\int_{s}^{1 / 2} q_{j}(t) d t\right) d s\right. & \\
& \left.+\int_{1 / 2}^{1-\delta} G\left(\int_{1 / 2}^{s} q_{j}(t) d t\right) d s, j=1, \ldots, m\right\}>0 .
\end{aligned}
$$

In the sequel, $\delta$ always satisfies (3.3).

We define a continuous and positive function on $[\delta, 1-\delta]$,

$$
z(x):=\min \left\{z_{j}(x): j=1, \ldots, m\right\}, \quad \delta \leq x \leq 1-\delta,
$$

where

$$
z_{j}(x):=\int_{\delta}^{x} G\left(\int_{s}^{x} q_{j}(t) d t\right) d s+\int_{x}^{1-\delta} G\left(\int_{x}^{s} q_{j}(t) d t\right) d s .
$$

Moreover, $M>0$ is chosen so that

$$
\delta^{2} \alpha M>2, \quad \alpha:=\min \{z(x): \delta \leq x \leq 1-\delta\}>0 .
$$

Further, since $f_{\infty}=\infty$, there exists $\varrho_{2}>\varrho_{1}$ such that

$$
f_{j}(u) \geq(M|u|)^{p-1} \quad \text { whenever }|u|>\delta^{2} \varrho_{2}, j=1, \ldots, m,
$$

where the constant $M$ satisfies (3.4).

Let $\Omega_{2}:=\left\{y \in E:\|y\|<\varrho_{2}\right\}$. Then each $y \in K_{\mathrm{a}}$ with $\|y\|=\varrho_{2}$ satisfies

$$
y_{j}(t) \geq \delta^{2} \varrho_{2} \quad \text { on }[\delta, 1-\delta] \text { for some } j \in\{1, \ldots, m\} .
$$


Consequently, it follows from (3.3)-(3.6) that for each $j \in\{1, \ldots, m\}$ satisfying (3.6) we have

$$
\begin{aligned}
2\left\|w_{j}\right\| & \geq \int_{\delta}^{\sigma_{j}} G\left(\int_{s}^{\sigma_{j}} q_{j}(t) f_{j}(y(t)) d t\right) d s+\int_{\sigma_{j}}^{1-\delta} G\left(\int_{\sigma_{j}}^{s} q_{j}(t) f_{j}(y(t)) d t\right) d s \\
& \geq \delta^{2} M \varrho_{2}\left\{\int_{\delta}^{\sigma_{j}} G\left(\int_{s}^{\sigma_{j}} q_{j}(t) d t\right) d s+\int_{\sigma_{j}}^{1-\delta} G\left(\int_{\sigma_{j}}^{s} q_{j}(t) d t\right) d s\right\} \\
& \geq \delta^{2} M \varrho_{2} \alpha>2 \varrho_{2}=2\|y\| \quad \text { when } \sigma_{j} \in[\delta, 1-\delta], \\
\left\|w_{j}\right\| & \geq \int_{\delta}^{1-\delta} G\left(\int_{s}^{1-\delta} q_{j}(t) f_{j}(y(t)) d t\right) d s \\
& \geq \delta^{2} M \varrho_{2} \alpha>\varrho_{2}=\|y\| \quad \text { when } \sigma_{j}>1-\delta, \\
\left\|w_{j}\right\| & \geq \int_{\delta}^{1-\delta} G\left(\int_{\delta}^{s} q_{j}(t) f_{j}(y(t)) d t\right) d s \\
& \geq \delta^{2} M \varrho_{2} \alpha>\|y\| \quad \text { when } \sigma_{j}<\delta .
\end{aligned}
$$

This shows that

$$
\left\|\Phi_{a} y\right\| \geq\left\|w_{j}\right\|>\|y\| \quad \forall y \in K_{\mathrm{a}} \cap \partial \Omega_{2} .
$$

Therefore, from the first part of Theorem 2 , we conclude that $\Phi_{\mathrm{a}}$ has a fixed point $y \in K_{\mathrm{a}} \cap\left(\bar{\Omega}_{2} \backslash \Omega_{1}\right)$. Since $0<\varrho_{1} \leq\|y\| \leq \varrho_{2}$, we see that $y(t)$ is a nontrivial nonnegative solution to the problem (1.7)-(1.8) a

Next consider $f_{0}=\infty$ and $f_{\infty}=0$. Since $f_{0}=\infty$, we may choose $\varrho_{1}>0$ such that

$$
f_{j}(u) \geq(M|u|)^{p-1} \quad \text { whenever } 0 \leq|u| \leq \varrho_{1}, j=1, \ldots, m,
$$

where the constant $M$ satisfies (3.4). Thus, each $y \in K_{\mathrm{a}}$ with $\|y\|=\varrho_{1}$ satisfies

$$
y_{j}(t) \geq \delta^{2} \varrho_{1} \quad \text { on }[\delta, 1-\delta] \text { for some } j \in\{1, \ldots, m\} .
$$

Then for each $j \in\{1, \ldots, m\}$ satisfying (3.7), in the same way as above, we can prove

$$
\left\|\Phi_{\mathrm{a}} y\right\| \geq\left\|w_{j}\right\|>\|y\| \quad \forall y \in K_{\mathrm{a}} \cap \partial \Omega_{1},
$$

where $\Omega_{1}:=\left\{y \in E:\|y\|<\varrho_{1}\right\}$.

Further, since $f_{\infty}=0$, there exists an $N>\varrho_{1}$ such that

$$
f_{j}(u) \leq\left(\frac{\varepsilon}{2}|u|\right)^{p-1} \quad \text { whenever }|u| \geq N, j=1, \ldots, m,
$$


where the constant $\varepsilon$ satisfies (3.2). Let

$$
\begin{aligned}
\frac{1}{2} \varrho_{2}> & N+\max \left\{f_{j}(u): 0 \leq|u| \leq N\right\}\left(\int_{0}^{1 / 2} G\left(\int_{s}^{1 / 2} q_{j}(t) d t\right) d s\right. \\
& \left.+\int_{1 / 2}^{1} G\left(\int_{1 / 2}^{s} q_{j}(t) d t\right) d s\right) \quad \text { for } j=1, \ldots, m .
\end{aligned}
$$

Then for $y \in K_{\mathrm{a}}$ with $\|y\|=\varrho_{2}$, for each $j \in\{1, \ldots, m\}$ we have

$$
\begin{aligned}
\left\|w_{j}\right\|= & \int_{0}^{\sigma_{j}} G\left(\int_{s}^{\sigma_{j}} q_{j}(t) f_{j}(y(t)) d t\right) d s \\
\leq & \int_{0}^{1 / 2} G\left(\int_{s}^{1 / 2} q_{j}(t) f_{j}(y(t)) d t\right) d s \\
< & N+\max \left\{f_{j}(u): 0 \leq|u| \leq N\right\}\left(\int_{0}^{1 / 2} G\left(\int_{s}^{1 / 2} q_{j}(t) d t\right) d s\right. \\
& \left.+\frac{\varepsilon}{2}\|y\| \int_{0}^{1 / 2} G\left(\int_{s}^{1 / 2} q_{j}(t) d t\right) d s\right) \\
< & \frac{1}{2} \varrho_{2}+\frac{1}{2}\|y\|=\|y\| \quad \text { when } \sigma_{j} \in(0,1 / 2] .
\end{aligned}
$$

When $\sigma_{j} \in[1 / 2,1)$, in the same way as above we have

$$
\left\|w_{j}\right\|<\|y\| \text {. }
$$

Hence we obtain

$$
\left\|\Phi_{\mathrm{a}} y\right\|<\|y\| \quad \forall y \in K_{\mathrm{a}} \cap \partial \Omega_{2},
$$

where $\Omega_{2}:=\left\{y \in E:\|y\|<\varrho_{2}\right\}$.

Therefore, by the second part of Theorem 2, it follows that $\Phi_{\mathrm{a}}$ has a fixed point $y(t)$ in $K_{\mathrm{a}} \cap\left(\bar{\Omega}_{2} \backslash \Omega_{1}\right)$, which is a nontrivial nonnegative solution to the problem $(1.7)-(1.8)_{\mathrm{a}}$.

Finally, we consider the problems $(1.7)-(1.8)_{b}$ and $(1.7)-(1.8)_{c}$. It is easy to check that they are equivalent to the integral equation

$$
y_{j}(t)=\int_{0}^{t} G\left(\int_{s}^{1} q_{j}(r) f_{j}(y(r)) d r\right) d s, \quad j=1, \ldots, m,
$$

and

$$
y_{j}(t)=\int_{t}^{1} G\left(\int_{0}^{s} q_{j}(r) f_{j}(y(r)) d r\right) d s, \quad j=1, \ldots, m,
$$

respectively, where $y(t)=\left(y_{1}(t), \ldots, y_{m}(t)\right)$. 
For the problem $(1.7)-(1.8)_{\mathrm{b}}$, let $K_{\mathrm{b}}$ be the cone given by $K_{\mathrm{b}}=\left\{y \in E: y_{j}(t)\right.$ is concave on $[0,1]$,

$$
\left.y_{j}(0)=0 \text { is the minimum and } y_{j}(1) \text { the maximum, } j=1, \ldots, m\right\} \text {. }
$$

For the problem $(1.7)-(1.8)_{\mathrm{c}}$, let $K_{\mathrm{c}}$ be the cone given by $K_{\mathrm{c}}=\left\{y \in E: y_{j}(t)\right.$ is concave on $[0,1]$,

$$
\left.y_{j}(0) \text { is the maximum and } y_{j}(1)=0 \text { the minimum, } j=1, \ldots, m\right\} \text {. }
$$

By the method above, Theorem 2 is employed to prove that $(1.7)-(1.8)_{\mathrm{b}}$ and $(1.7)-(1.8)_{c}$ have nontrivial nonnegative solutions. This completes the proof of Theorem 1.

Acknowledgements. The authors thank the referee for his valuable suggestions.

\section{References}

[1] L. H. Erbe and H. Y. Wang, On the existence of positive solutions of ordinary differential equations, Proc. Amer. Math. Soc. 120 (1994), 743-748.

[2] R. Y. Ma, Existence of positive radial solutions for elliptic systems, J. Math. Anal. Appl. 201 (1996), 375-386.

Department of Mathematics Northeast Normal University Changchun 130024, China E-mail: sxxi@nenu.edu.cn

\author{
Department of Mathematics \\ Harbin Normal University \\ Harbin 150080, China
}

